\title{
Uniform propagation of chaos for Kac's 1D particle system
}

\author{
Roberto Cortez*
}

October 3, 2018

\begin{abstract}
In this paper we study Kac's 1D particle system, consisting of the velocities of $N$ particles colliding at constant rate and randomly exchanging energies. We prove uniform (in time) propagation of chaos in Wasserstein distance with explicit polynomial rates in $N$, for both the squared (i.e., the energy) and non-squared particle system. These rates are of order $N^{-1 / 3}$ (almost, in the non-squared case), assuming that the initial distribution of the limit nonlinear equation has finite moments of sufficiently high order $(4+\epsilon$ is enough when using the 2-Wasserstein distance). The proof relies on a convenient parametrization of the collision recently introduced by Hauray, as well as on a coupling technique developed by Cortez and Fontbona.
\end{abstract}

Keywords: Kinetic theory, Kac particle system, Propagation of chaos.

MSC 2010: 82C40, 60K35.

\section{Introduction and main results}

In this paper we study Kac's particle system, introduced in [9] and later studied for instance in [2, 3, 4, 5]. It can be described as follows: consider $N$ objects or "particles" characterized by their one-dimensional velocities, subjected to the following binary random "collisions": when particles with velocities $v$ and $v_{*}$ collide, they acquire new velocities $v^{\prime}$ and $v_{*}^{\prime}$ given by the rule

$$
\left(v, v_{*}\right) \mapsto\left(v^{\prime}, v_{*}^{\prime}\right)=\left(v \cos \theta-v_{*} \sin \theta, v_{*} \cos \theta+v \sin \theta\right),
$$

where $\theta \in[0,2 \pi)$ is chosen uniformly at random. This can be seen as a rotation in $\theta$ of the pair $\left(v, v_{*}\right) \in \mathbb{R}^{2}$ and, as such, it preserves the energy, i.e., $v^{2}+v_{*}^{2}=v^{\prime 2}+v_{*}^{\prime 2}$. The system evolves continuously with time $t \geq 0$; the times between collisions follow an exponential law with parameter $N / 2$ and the two particles that collide are chosen randomly among all possible pairs, so each particle collides once per unit of time on average. The system starts at $t=0$ with some fixed symmetric distribution, and all the previous random

${ }^{*}$ CIMFAV, Facultad de Ingeniería, Universidad de Valparaíso. General Cruz 222, Valparaíso, Chile. E-mail: rcortez@dim.uchile.cl. Supported by Fondecyt Postdoctoral Project 3160250. 
choices are made independently. This description unambigously determines (the law of) the particle system, which we denote $\mathbf{V}_{t}=\left(V_{1, t}, \ldots, V_{N, t}\right)$.

In the pioneering work [9], Kac proved that for all $t \geq 0$, as $N \rightarrow \infty$, the empirical measure of the system $\frac{1}{N} \sum_{i} \delta_{V_{i, t}}$ converges weakly to $f_{t}$ (provided that the convergence holds for $t=0)$, where $\left(f_{t}\right)_{t \geq 0}$ is the collection of probability measures on $\mathbb{R}$ solving the so-called Boltzmann-Kac equation:

$$
\partial_{t} f_{t}(v)=\int_{0}^{2 \pi} \int_{\mathbb{R}}\left[f_{t}\left(v^{\prime}\right) f_{t}\left(v_{*}^{\prime}\right)-f_{t}(v) f_{t}\left(v_{*}\right)\right] d v_{*} \frac{d \theta}{2 \pi} .
$$

This convergence is now termed propagation of chaos, and it has been extensively studied during the last decades for this and other, more general kinetic models (especially the Boltzmann equation), see for instance [11, 10] and the references therein.

Another interesting feature of this model is its behaviour as $t \rightarrow \infty$. For instance, assuming normalized initial energy, i.e., $\sum_{i} V_{i, 0}^{2}=N$ a.s., it is known that the law of the system converges exponentially in $L^{2}$ to its equilibrium, namely, the uniform distribution on the Kac sphere $\left\{\mathbf{x} \in \mathbb{R}^{N}: \sum_{i} x_{i}^{2}=N\right\}$, see [3] and the references therein. As an alternative approach, one can couple two copies of the particle system using the same collision times and the same angle $\theta$ (i.e., "parallel coupling"), but with different initial conditions, to show that the 2-Wasserstein distance between their laws is non-increasing in time. However, a simple and better coupling was recently introduced in [8]: note first that the post-collisional velocities in (11) can be written as $\left(v^{\prime}, v_{*}^{\prime}\right)=\sqrt{v^{2}+v_{*}^{2}}(\cos (\alpha+$ $\theta), \sin (\alpha+\theta))$, where $\alpha \in(-\pi, \pi]$ is the angle defined by $\left(v, v_{*}\right)=\sqrt{v^{2}+v_{*}^{2}}(\cos \alpha, \sin \alpha)$, with the convention that all sums of angles are modulo $2 \pi$; next, note that, since $\theta$ is uniformly chosen in $[0,2 \pi)$, so is $\alpha+\theta$, and then the interaction rule

$$
\left(v, v_{*}\right) \mapsto\left(v^{\prime}, v_{*}^{\prime}\right)=\sqrt{v^{2}+v_{*}^{2}}(\cos (\theta), \sin (\theta))
$$

generates a system that has the same law than the one described by (11). Using this new parametrization of the collision, one can define a coupling that leads to contraction results in some Wasserstein metrics, see [8] for details.

Our goal in this paper is to use the parametrization (3) in a propagation of chaos context, in order to obtain explicit (in $N$ ) and uniform-in-time rates of convergence, as $N \rightarrow \infty$, for the law of the particles towards the solution of (2). We will quantify this convergence using the $p$-Wasserstein distance: given two probability measures $\mu$ and $\nu$ on $\mathbb{R}^{k}$, it is defined as

$$
\mathcal{W}_{p}(\mu, \nu)=\left(\inf \mathbb{E} \frac{1}{k} \sum_{i=1}^{k}\left|X_{i}-Y_{i}\right|^{p}\right)^{1 / p}
$$

where the infimum is taken over all random vectors $\mathbf{X}=\left(X_{1}, \ldots, X_{k}\right)$ and $\mathbf{Y}=\left(Y_{1}, \ldots, Y_{k}\right)$ such that $\mathcal{L}(\mathbf{X})=\mu$ and $\mathcal{L}(\mathbf{Y})=\nu$ (we do not specify the dependence on $k$ in our notation). We use the normalized distance $|\mathbf{x}-\mathbf{y}|_{k}^{p}=\frac{1}{k} \sum_{i}\left|x_{i}-y_{i}\right|^{p}$ on $\mathbb{R}^{k}$, which is natural when one cares about the dependence on the dimension. A pair $(\mathbf{X}, \mathbf{Y})$ attaining the infimum is called an optimal coupling and it can be shown that it always exists. See for instance [13] for background on optimal coupling and Wasserstein distances.

Let us fix some notation. We denote $E_{N}=\frac{1}{N} \sum_{i} V_{i, 0}^{2}$ the (random) mean initial energy, which is preserved, i.e., $\frac{1}{N} \sum_{i} V_{i, t}^{2}=E_{N}$ for all $t \geq 0$, a.s. We also denote $\mathcal{E}=\int_{\mathbb{R}} v^{2} f_{0}(d v)$, which itself is preserved by the flow $\left(f_{t}\right)_{t \geq 0}$. For a vector $\mathbf{x}=\left(x_{1}, \ldots, x_{N}\right) \in \mathbb{R}^{N}$ we 
denote by $\mathbf{x}^{(2)}=\left(x_{1}^{2}, \ldots, x_{N}^{2}\right)$ the vector of squares of $\mathbf{x}$, and we define the (empirical) probability measures $\overline{\mathbf{x}}=\frac{1}{N} \sum_{j} \delta_{x_{j}}$ and $\overline{\mathbf{x}}_{i}=\frac{1}{N-1} \sum_{j \neq i} \delta_{x_{j}}$. Also, for a probability measure $\mu$ on $\mathbb{R}$, we denote by $\mu^{(2)}$ the measure on $\mathbb{R}_{+}$defined by $\int \phi(v) \mu^{(2)}(d v)=\int \phi\left(v^{2}\right) \mu(d v)$.

Theorem 1. Assume that $\int_{\mathbb{R}}|v|^{p} f_{0}(d v)<\infty$ for some $p>4, p \neq 8$. Let $\gamma=\min \left(\frac{1}{3}, \frac{p-4}{2 p-4}\right)$ and $\lambda_{N}=\frac{1}{4} \frac{N+2}{N-1}$. Then, there exists a constant $C$ depending only on $p$ and $\int_{\mathbb{R}}|v|^{p} f_{0}(d v)$, such that for all $t \geq 0$,

$$
\begin{aligned}
\mathbb{E} \mathcal{W}_{2}^{2}\left(\overline{\mathbf{V}}_{t}^{(2)}, f_{t}^{(2)}\right) \leq & \frac{C}{N^{\gamma}}+C \mathbb{E}\left(E_{N}-\mathcal{E}\right)^{2} \\
& +C e^{-\lambda_{N} t} \mathcal{W}_{2}^{2}\left(\mathcal{L}\left(\mathbf{V}_{0}^{(2)}\right),\left(f_{0}^{(2)}\right)^{\otimes N}\right)
\end{aligned}
$$

This yields a uniform-in-time propagation of chaos in $\mathcal{W}_{2}^{2}$ for the energy of the particles. For instance, assuming that $\int|v|^{p} f_{0}(d v)<\infty$ for some $p>8$, the result gives a rate of order $N^{-1 / 3}$, provided that $\mathbb{E}\left(E_{N}-\mathcal{E}\right)^{2}$ and $\mathcal{W}_{2}^{2}\left(\mathcal{L}\left(\mathbf{V}_{0}^{(2)}\right),\left(f_{0}^{(2)}\right)^{\otimes N}\right)$ converge to 0 at the same rate or faster. Notice also that $\lambda_{N}$ coincides with the spectral gap in $L^{2}$ of the associated generator of the particle system, which was computed in [3] (although with a factor 2 due to a different rate of the collision times). The restriction $p \neq 8$ comes from the fact that the proof of Theorem 1 makes use of a general chaocity result for i.i.d. sequences found in [7, Theorem 1]; including the case $p=8$ would produce additional logarithmic terms in the rate, see (15) below.

As in [8, Corollary 3], this $\mathcal{W}_{2}^{2}$ propagation of chaos result for the energy implies the following $\mathcal{W}_{4}^{4}$ result for the non-squared system:

Corollary 2. Let $\mathbf{U}_{0}=\left(U_{1,0}, \ldots, U_{N, 0}\right)$ be any vector of i.i.d. and $f_{0}$-distributed random variables, and let $\tilde{\gamma}=\frac{p-4}{2 p} \mathbf{1}_{p<8}+\frac{p-4}{3 p-8} \mathbf{1}_{p>8}$. Under the same assumptions as in Theorem 1. we have for all $t \geq 0$,

$$
\begin{gathered}
\mathbb{E} \mathcal{W}_{4}^{4}\left(\overline{\mathbf{V}}_{t}, f_{t}\right) \leq \frac{C}{N_{\tilde{\gamma}}}+C \mathbb{E}\left(E_{N}-\mathcal{E}\right)^{2}+C e^{-\lambda_{N} t} \mathbb{E}\left[\frac{1}{N} \sum_{i=1}^{N}\left(V_{i, 0}^{2}-U_{i, 0}^{2}\right)^{2}\right] \\
+C e^{-t} \mathbb{E}\left[\frac{1}{N} \sum_{i=1}^{N}\left(V_{i, 0}-U_{i, 0}\right)^{4}\right] .
\end{gathered}
$$

Notice that $\tilde{\gamma}<\gamma$ for all $p>4$, thus the rate obtained is slower than the one of Theorem 1 (although we can easily deduce a rate $N^{-\gamma}$ in $\mathcal{W}_{4}^{4}$ for the law of one particle). For instance, if $f_{0}$ has finite moment of order $p>8$, Corollary 2 gives a chaos rate of $N^{-1 / 4}$ in $\mathcal{W}_{4}^{4}$; but if $f_{0}$ has finite moments of all orders, it yields a rate of almost $N^{-1 / 3}$.

Note that when $p$ is close to 4 , the chaos rates provided by these results are very slow. The following theorem provides a good rate assuming only that $f_{0}$ has finite moment of order $4+\epsilon$ :

Theorem 3. Assume that $\int_{\mathbb{R}}|v|^{p} f_{0}(d v)<\infty$ for some $p>4$, and that $\sup _{N} \mathbb{E} V_{1,0}^{4}<\infty$. Then, there exists a constant $C$ depending only on $p$, on $\int_{\mathbb{R}}|v|^{p} f_{0}(d v)$ and on $\sup _{N} \mathbb{E} V_{1,0}^{4}$, such that for all $t \geq 0$,

$$
\mathbb{E} \mathcal{W}_{2}^{2}\left(\overline{\mathbf{V}}_{t}, f_{t}\right) \leq \frac{C \log ^{2} N}{N^{1 / 3}}+C \mathcal{W}_{2}^{2}\left(\mathcal{L}\left(\mathbf{V}_{0}\right), f_{0}^{\otimes N}\right)
$$

To the best of our knowledge, these are the first uniform propagation of chaos results for Kac's 1D particle system; they will be proven in Section 3. Similar results for the law 
of $k$ particles can also be stated. The rates are explicit and of order $N^{-1 / 3}$ (almost, in Corollary 2 and Theorem 3), assuming enough moments of $f_{0}$. This is quite reasonable, given that in general the optimal rate of chaocity for an i.i.d. sequence is $N^{-1 / 2}$, see [7, Theorem 1]. Notice that in these results, the initial condition $\mathbf{V}_{0}$ is not restricted to have fixed (non-random) mean energy, and can thus be chosen at convenience. For instance, it can have distribution $f_{0}^{\otimes N}$, thus the term $\mathbb{E}\left(E_{N}-\mathcal{E}\right)^{2}$ in Theorem 1 and Corollary 2 is easily seen to be of order $1 / N$, while the terms $\mathcal{W}_{2}^{2}\left(\mathcal{L}\left(\mathbf{V}_{0}^{(2)}\right),\left(f_{0}^{(2)}\right)^{\otimes N}\right), \sum_{i}\left(V_{i, 0}^{2}-U_{i, 0}^{2}\right)^{2}$, $\sum_{i}\left(V_{i, 0}-U_{i, 0}\right)^{4}$ and $\mathcal{W}_{2}^{2}\left(\mathcal{L}\left(\mathbf{V}_{0}\right), f_{0}^{\otimes N}\right)$ all vanish. Or one can assume normalized energy (i.e., $E_{N}=\mathcal{E}$ a.s.), provided that one can control the remaining terms.

We remark that, although one could use the general functional techniques of [10] in the present context, the rates obtained with these techniques are likely to be much slower than the ones presented here.

The proof of our results mainly relies on the parametrization (3) introduced in [8], and on a coupling argument developed in [6] to relate the behaviour of the particle system and the limit jump process (the nonlinear process). We remark however that, while the proof of Theorem [1 makes use of the techniques of [8] and [6], the proof of Theorem [3] directly combines the results found in these references.

\section{Construction}

We now give a specific construction of the particle system and couple it with a suitable system of nonlinear processes, following [6]. Consider a Poisson point measure $\mathcal{N}(d t, d \theta, d \xi, d \zeta)$ on $\mathbb{R}_{+} \times[0,2 \pi) \times[0, N) \times[0, N)$ with intensity $\frac{d t d \theta d \xi d \zeta \mathbf{1}_{\mathcal{G}}(\xi, \zeta)}{4 \pi(N-1)}$, where $\mathcal{G}:=\left\{(\xi, \zeta) \in[0, N)^{2}: \mathbf{i}(\xi) \neq \mathbf{i}(\zeta)\right\}$ and $\mathbf{i}(\xi):=\lfloor\xi\rfloor+1$. In words, the measure $\mathcal{N}$ picks collision times $t \in \mathbb{R}_{+}$at rate $N / 2$, and for each such $t$, it also independently samples an angle $\theta$ uniformly at random from $[0,2 \pi)$ and a pair $(\xi, \zeta)$ uniformly from the set $\mathcal{G}$ (note that the area of $\mathcal{G}$ is $N(N-1))$. The pair $(\mathbf{i}(\xi), \mathbf{i}(\zeta))$ gives the indices of the particles that jump at each collision. Using the parametrization (3), we define the particle system $\mathbf{V}_{t}=\left(V_{1, t}, \ldots, V_{N, t}\right)$ as the solution to

$$
d V_{i, t}=\int_{0}^{2 \pi} \int_{A_{i}}\left[\sqrt{V_{i, t^{-}}^{2}+V_{\mathbf{i}(\xi), t^{-}}^{2}} \cos \theta-V_{i, t^{-}}\right] \mathcal{N}_{i}(d t, d \theta, d \xi),
$$

for all $i \in\{1, \ldots, N\}$, where $A_{i}:=[0, N) \backslash[i-1, i)$, and $\mathcal{N}_{i}$ is the point measure defined as

$$
\mathcal{N}_{i}(d t, d \theta, d \xi)=\mathcal{N}(d t, d \theta,[i-1, i), d \xi)+\mathcal{N}(d t, d \theta-\pi / 2, d \xi,[i-1, i))
$$

where the $-\pi / 2$ is to transform sinus to cosinus. Clearly, $\mathcal{N}_{i}$ is a Poisson point measure on $\mathbb{R}_{+} \times[0,2 \pi) \times A_{i}$ with intensity $\frac{d t d \theta d \xi}{2 \pi(N-1)}$. The initial condition $\mathbf{V}_{0}=\left(V_{1,0}, \ldots, V_{N, 0}\right)$ is some random vector with exchangeable components, independent of $\mathcal{N}$.

The nonlinear process (introduced by Tanaka [12] in the context of the Boltzmann equation for Maxwell molecules) is a stochastic jump-process having marginal laws $\left(f_{t}\right)_{t \geq 0}$, and it is the probabilistic counterpart of (2). It represents the trajectory of a fixed particle inmersed in the infinite population, and it is obtained, for instance, as the solution to (44) when one replaces $V_{\mathbf{i}(\xi), t^{-}}$(which is a $\xi$-realization of the (random) measure $\overline{\mathbf{V}}_{i, t^{-}}=$ $\left.\frac{1}{N-1} \sum_{j \neq i} \delta_{V_{j, t^{-}}}\right)$with a realization of $f_{t}$.

The key idea, introduced in [6], is to define, for each $i \in\{1, \ldots, N\}$, a nonlinear process $U_{i, t}$ that mimics as closely as possible the dynamics of $V_{i, t}$, which is achieved 
using a suitable realization of $f_{t}$ at each collision. More specifically: the collection $\mathbf{U}_{t}=$ $\left(U_{1, t}, \ldots, U_{N, t}\right)$ is defined as the solution to

$$
d U_{i, t}=\int_{0}^{2 \pi} \int_{A_{i}}\left[\sqrt{U_{i, t^{-}}^{2}+F_{i, t}^{2}\left(\mathbf{U}_{t^{-}}, \xi\right)} \cos \theta-U_{i, t^{-}}\right] \mathcal{N}_{i}(d t, d \theta, d \xi),
$$

for all $i \in\{1, \ldots, N\}$. Here, $F_{i}$ is a measurable mapping $\mathbb{R}_{+} \times \mathbb{R}^{N} \times A_{i} \ni(t, \mathbf{x}, \xi) \mapsto$ $F_{i, t}(\mathbf{x}, \xi)$ such that for all $(t, \mathbf{x})$ and any random variable $\xi$ which is uniformly distributed on $A_{i}$, the pair $\left(x_{\mathbf{i}(\xi)}, F_{i, t}(\mathbf{x}, \xi)\right)$ is an optimal coupling between $\overline{\mathbf{x}}_{i}=\frac{1}{N-1} \sum_{j \neq i} \delta_{x_{j}}$ and $f_{t}$ with respect to the cost function $c(x, y)=\left(x^{2}-y^{2}\right)^{2}$. Thus,

$$
\int_{A_{i}}\left(x_{\mathbf{i}(\xi)}^{2}-F_{i, t}^{2}(\mathbf{x}, \xi)\right)^{2} \frac{d \xi}{N-1}=\mathcal{W}_{2}^{2}\left(\overline{\mathbf{x}}_{i}^{(2)}, f_{t}^{(2)}\right) \text {. }
$$

We refer to [6, Lemma 3] for a proof of existence of such a mapping (here we use a different cost, but our proof works for any cost that is continuous and bounded from below, in order to use a measurable selection result of optimal transference plans, such as [13, Corollary 5.22]). That lemma also shows that for any $i \neq j \in\{1, \ldots, N\}$, any random vector $\mathbf{X} \in \mathbb{R}^{N}$ with exchangeable components and any bounded and Borel measurable $\phi: \mathbb{R} \rightarrow \mathbb{R}$, we have

$$
\mathbb{E} \int_{i-1}^{i} \phi\left(F_{i, t}(\mathbf{X}, \xi)\right) d \xi=\int_{\mathbb{R}} \phi(v) f_{t}(d v) .
$$

The initial conditions $U_{1,0}, \ldots, U_{N, 0}$ are taken independently and with law $f_{0}$. For instance, they can be chosen such that the pair $\left(\mathbf{V}_{0}, \mathbf{U}_{0}\right)$ is an optimal coupling between $\mathcal{L}\left(\mathbf{V}_{0}\right)$ and $f_{0}^{\otimes N}$ with respect to the cost function $\left(x^{2}-y^{2}\right)^{2}$, so that $\mathbb{E} \frac{1}{N} \sum_{i}\left(V_{i, 0}^{2}-U_{i, 0}^{2}\right)^{2}=$ $\mathcal{W}_{2}^{2}\left(\mathcal{L}\left(\mathbf{V}_{0}^{(2)}\right),\left(f_{0}^{(2)}\right)^{\otimes N}\right)$ (this is done in the proof of Theorem 1, but, in general, $\mathbf{U}_{0}$ can be any random vector with law $\left.f_{0}^{\otimes N}\right)$.

Strong existence and uniqueness of solutions $\mathbf{V}_{t}=\left(V_{1, t}, \ldots, V_{N, t}\right)$ and $\mathbf{U}_{t}=\left(U_{1, t}, \ldots, U_{N, t}\right)$ for (44) and (6) are straightforward: since the total rate of $\mathcal{N}$ is finite over finite time intervals, those equations are nothing but recursions for the values of the processes at the (timely ordered) jump times. Also, the collection of pairs $\left(V_{1}, U_{1}\right), \ldots,\left(V_{N}, U_{N}\right)$ is clearly exchangeable.

Every $U_{i, t}$ is a nonlinear process, thus $\mathcal{L}\left(U_{i, t}\right)=f_{t}$ for all $t$. Note however that $U_{i, t}$ and $U_{j, t}$ have simultaneous jumps, and consequently they are not independent. As in [6], in order to obtain the desired results, we will need to show that they become asymptotically independent as $N \rightarrow \infty$, which is achieved using a second coupling, see Lemma 6 below.

\section{Proofs}

We will need the following propagation of moments result.

Lemma 4. Assume that $\int_{\mathbb{R}}|v|^{p} f_{0}(d v)<\infty$ for some $p \geq 2$. Then there exists $C>0$ depending only on $p$ and $\int_{\mathbb{R}}|v|^{p} f_{0}(d v)$ such that $\int_{\mathbb{R}}|v|^{p} f_{t}(d v)<C$ for all $t \geq 0$.

Proof. See the proof of [6, Lemma 5].

Lemma 5. Assume that $\int_{\mathbb{R}} v^{4} f_{0}(d v)<\infty$. Then, there exists a constant $C$ depending only on $\int_{\mathbb{R}} v^{4} f_{0}(d v)$, such that for any $i \neq j$,

$$
\left|\operatorname{cov}\left(U_{i, t}^{2}, U_{j, t}^{2}\right)\right| \leq\left(1-e^{-t}\right) \frac{C}{N} .
$$


Proof. We will estimate $h_{t}:=\mathbb{E}\left(U_{i, t}^{2} U_{j, t}^{2}\right)$. From (66) we have

$$
\begin{aligned}
d h_{t}=\mathbb{E} \int_{0}^{2 \pi} & \int_{[0, N)^{2}}\left[\mathbf{1}_{\{\mathbf{i}(\xi)=i, \mathbf{i}(\zeta)=j\}} \Delta_{1}+\mathbf{1}_{\{\mathbf{i}(\xi)=j, \mathbf{i}(\zeta)=i\}} \Delta_{2}\right. \\
& +\mathbf{1}_{\{\mathbf{i}(\xi)=i, \mathbf{i}(\zeta) \neq j\}} \Delta_{3}+\mathbf{1}_{\{\mathbf{i}(\xi) \neq j, \mathbf{i}(\zeta)=i\}} \Delta_{4} \\
& \left.+\mathbf{1}_{\{\mathbf{i}(\xi) \neq i, \mathbf{i}(\zeta)=j\}} \Delta_{5}+\mathbf{1}_{\{\mathbf{i}(\xi)=j, \mathbf{i}(\zeta) \neq i\}} \Delta_{6}\right] \mathcal{N}(d t, d \theta, d \xi, d \zeta)
\end{aligned}
$$

where $\Delta_{1}$ and $\Delta_{2}$ are the increments of $U_{i, t}^{2} U_{j, t}^{2}$ when $U_{i, t}$ and $U_{j, t}$ have a simultanous jump, and $\Delta_{3}, \ldots, \Delta_{6}$ are the increments when only one of them jumps. For instance,

$$
\begin{aligned}
& \Delta_{1}=\left(U_{i, t^{-}}^{2}+F_{i, t}^{2}\left(\mathbf{U}_{t^{-}}, \zeta\right)\right) \cos ^{2} \theta\left(U_{j, t^{-}}^{2}+F_{j, t}^{2}\left(\mathbf{U}_{t^{-}}, \xi\right)\right) \sin ^{2} \theta-U_{i, t^{-}}^{2} U_{j, t^{-}}^{2}, \\
& \Delta_{3}=\left(U_{i, t^{-}}^{2}+F_{i, t}^{2}\left(\mathbf{U}_{t^{-}}, \zeta\right)\right) \cos ^{2} \theta U_{j, t^{-}}^{2}-U_{i, t^{-}}^{2} U_{j, t^{-}}^{2}
\end{aligned}
$$

We have for the latter:

$$
\begin{aligned}
& \mathbb{E} \int_{0}^{2 \pi} \int_{[0, N)^{2}} \mathbf{1}_{\{\mathbf{i}(\xi)=i, \mathbf{i}(\zeta) \neq j\}} \Delta_{3} \mathcal{N}(d t, d \theta, d \xi, d \zeta) \\
& =\mathbb{E} \int_{0}^{2 \pi} \int_{A_{i}}\left[-\left(1-\cos ^{2} \theta\right) U_{i, t}^{2} U_{j, t}^{2}\right. \\
& \left.\quad+\cos ^{2} \theta F_{i, t}^{2}\left(\mathbf{U}_{t}, \zeta\right) U_{j, t}^{2}\right] \frac{d t d \theta d \zeta}{4 \pi(N-1)} \\
& =\left[-\frac{1}{4} h_{t}+\frac{1}{4} \mathcal{E}^{2}\right] d t
\end{aligned}
$$

where we have used that $U_{j, t} \sim f_{t}$ under $\mathbb{P}$ and $F_{i, t}\left(\mathbf{U}_{t}, \zeta\right) \sim f_{t}$ under $\frac{d \zeta \mathbf{1}_{A_{i}}(\zeta)}{N-1}$. The same identity holds for $\Delta_{4}, \Delta_{5}$ and $\Delta_{6}$. On the other hand for $\Delta_{1}$ we can simply use the Cauchy-Schwarz inequality and the fact that $\mathbb{E} \int_{j-1}^{j} F_{i, t}^{4}\left(\mathbf{U}_{t}, \zeta\right) d \zeta=\int v^{4} f_{t}(d v) \leq C$ (thanks to (8) and Lemma 4), thus obtaining

$$
-\frac{C}{N} d t \leq \mathbb{E} \int_{0}^{2 \pi} \int_{[0, N)^{2}} \mathbf{1}_{\{\mathbf{i}(\xi)=i, \mathbf{i}(\zeta)=j\}} \Delta_{1} \mathcal{N}(d t, d \theta, d \xi, d \zeta) \leq \frac{C}{N} d t .
$$

The same estimate holds true for $\Delta_{2}$. Using this and (10) in (9), we deduce that $-h_{t}+\mathcal{E}^{2}-$ $\frac{C}{N} \leq \partial_{t} h_{t} \leq-h_{t}+\mathcal{E}^{2}+\frac{C}{N}$, and multiplying by $e^{t}$ and integrating yields $\left(e^{t}-1\right)\left(\mathcal{E}^{2}-\frac{C}{N}\right) \leq$ $e^{t} h_{t}-h_{0} \leq\left(e^{t}-1\right)\left(\mathcal{E}^{2}+\frac{C}{N}\right)$. But $U_{i, 0}$ and $U_{j, 0}$ are independent, thus $h_{0}=\mathcal{E}^{2}$, and then $\mathcal{E}^{2}-\left(1-e^{-t}\right) \frac{C}{N} \leq h_{t} \leq \mathcal{E}^{2}+\left(1-e^{-t}\right) \frac{C}{N}$. Since $\operatorname{cov}\left(U_{i, t}^{2}, U_{j, t}^{2}\right)=h_{t}-\mathcal{E}^{2}$, the conclusion follows.

For a given exchangeable random vector $\mathbf{X}$ on $\mathbb{R}^{N}$, denote $\mathcal{L}^{n}(\mathbf{X})$ the joint law of its $n$ first components. The following lemma provides a decoupling property for the system of nonlinear processes $\mathbf{U}_{t}$.

Lemma 6. Assume $\int_{\mathbb{R}} v^{4} f_{0}(d v)<\infty$. Then there exists a constant $C>0$, depending only on $\int_{\mathbb{R}} v^{4} f_{0}(d v)$, such that for all $n \leq N$ and $t \geq 0$,

$$
\mathcal{W}_{2}^{2}\left(\mathcal{L}^{n}\left(\mathbf{U}_{t}^{(2)}\right),\left(f_{t}^{(2)}\right)^{\otimes n}\right) \leq C \frac{n}{N}
$$

Also, if $\int_{\mathbb{R}}|v|^{p} f_{0}(d v)<\infty$ for some $p>4$, then there exists a constant $C>0$, depending only on $p$ and $\int_{\mathbb{R}}|v|^{p} f_{0}(d v)$, such that for all $n \leq N$ and $t \geq 0$,

$$
\mathcal{W}_{4}^{4}\left(\mathcal{L}^{n}\left(\mathbf{U}_{t}\right), f_{t}^{\otimes n}\right) \leq C\left(\frac{n}{N}\right)^{\frac{p-4}{p}}
$$


Proof. The argument uses a coupling construction, as in the proof of [6, Lemma 6]. We repeat the important steps here. First, for all $n \in\{2, \ldots, N\}$, the idea is to construct $n$ independent nonlinear processes $\tilde{U}_{1, t}, \ldots, \tilde{U}_{n, t}$ such that $\tilde{U}_{i, t}$ remains close to $U_{i, t}$ on average. To achieve this, let $\mathcal{M}$ be an independent copy of the Poisson point measure $\mathcal{N}$, and define for all $i \in\{1, \ldots, n\}$

$$
\begin{aligned}
\mathcal{M}_{i}(d t, d \theta, d \xi)=\mathcal{N} & (d t, d \theta,[i-1, i), d \xi) \\
& +\mathcal{N}(d t, d \theta-\pi / 2, d \xi,[i-1, i)) \mathbf{1}_{[n, N)}(\xi) \\
& +\mathcal{M}(d t, d \theta-\pi / 2, d \xi,[i-1, i)) \mathbf{1}_{[0, n)}(\xi)
\end{aligned}
$$

which is a Poisson point measure on $\mathbb{R}_{+} \times[0,2 \pi) \times A_{i}$ with intensity $\frac{d t d \theta d \xi}{2 \pi(N-1)}$, just as $\mathcal{N}_{i}$. We then define $\tilde{U}_{i, t}$ starting with $\tilde{U}_{i, 0}=U_{i, 0}$ and solving an equation similar to (6), but using $\mathcal{M}_{i}$ in place of $\mathcal{N}_{i}$ :

$$
d \tilde{U}_{i, t}=\int_{0}^{2 \pi} \int_{A_{i}}\left[\sqrt{\tilde{U}_{i, t^{-}}^{2}+F_{i, t}^{2}\left(\mathbf{U}_{t^{-}}, \xi\right)} \cos \theta-\tilde{U}_{i, t^{-}}\right] \mathcal{M}_{i}(d t, d \theta, d \xi) .
$$

In words, the processes $\tilde{U}_{1, t}, \ldots, \tilde{U}_{n, t}$ use the same atoms of $\mathcal{N}$ that $U_{1, t}, \ldots, U_{n, t}$ use, except for those that produce a joint jump of $U_{i, t}$ and $U_{j, t}$ for some $i, j \in\{1, \ldots, n\}$, in which case either $\tilde{U}_{i, t}$ or $\tilde{U}_{j, i}$ does not jump at that instant. To compensate for the missing jumps, additional independent atoms, drawn from $\mathcal{M}$, are added to $\mathcal{M}_{i}$.

It is clear that $\mathcal{M}_{1}, \ldots, \mathcal{M}_{n}$ are independent Poisson point measures. Using this and the fact that $F_{i, t}(\mathbf{x}, \xi)$ has distribution $f_{t}$ when $\xi$ is uniformly distributed on $A_{i}$, one can show that $\tilde{U}_{1, t}, \ldots, \tilde{U}_{n, t}$ are independent nonlinear processes; see the details in the proof of [6, Lemma 6].

Thus, $\mathcal{W}_{2}^{2}\left(\mathcal{L}^{n}\left(\mathbf{U}_{t}^{(2)}\right),\left(f_{t}^{(2)}\right)^{\otimes n}\right) \leq \mathbb{E} \frac{1}{n} \sum_{i=1}^{n}\left(U_{i, t}^{2}-\tilde{U}_{i, t}^{2}\right)^{2}$, and then, to deduce the first bound, it suffices to estimate $h_{t}:=\mathbb{E}\left(U_{i, t}^{2}-\tilde{U}_{i, t}^{2}\right)^{2}$ for any fixed $i \in\{1, \ldots, n\}$. From (6) and (12) we have

$$
\begin{aligned}
d h_{t}=\mathbb{E} \int_{0}^{2 \pi} \int_{A_{i}} \Delta_{1}[\mathcal{N}(d t, d \theta,[i-1, i), d \xi) & \left.+\mathcal{N}(d t, d \theta-\pi / 2, d \xi,[i-1, i)) \mathbf{1}_{[n, N)}(\xi)\right] \\
+ & \mathbb{E} \int_{0}^{2 \pi} \int_{A_{i}} \Delta_{2} \mathcal{N}(d t, d \theta-\pi / 2, d \xi,[i-1, i)) \mathbf{1}_{[0, n)}(\xi) \\
& +\mathbb{E} \int_{0}^{2 \pi} \int_{A_{i}} \Delta_{3} \mathcal{M}(d t, d \theta-\pi / 2, d \xi,[i-1, i)) \mathbf{1}_{[0, n)}(\xi)
\end{aligned}
$$

where $\Delta_{1}$ is the increment of $\left(U_{i, t}^{2}-\tilde{U}_{i, t}^{2}\right)^{2}$ when $U_{i, t}$ and $\tilde{U}_{i, t}$ have a simultaneous jump, $\Delta_{2}$ is the increment when only $U_{i, t}$ jumps, and $\Delta_{3}$ is the increment when only $\tilde{U}_{i, t}$ jumps. Thanks to the indicator $\mathbf{1}_{[0, n)}(\xi)$ and Lemma 4, the second and third terms in (13) are easily seen to be of order $C \frac{n}{N}$. For the first term, we have

$$
\begin{aligned}
& \Delta_{1}=\left(\left(U_{i, t^{-}}^{2}+F_{i, t}^{2}\left(\mathbf{U}_{t^{-}}, \xi\right)\right) \cos ^{2} \theta-\left(\tilde{U}_{i, t^{-}}^{2}+F_{i, t}^{2}\left(\mathbf{U}_{t^{-}}, \xi\right)\right) \cos ^{2} \theta\right)^{2} \\
&-\left(U_{i, t^{-}}^{2}-\tilde{U}_{i, t^{-}}^{2}\right)^{2} \\
&=-\left(1-\cos ^{4} \theta\right)\left(U_{i, t^{-}}^{2}-\tilde{U}_{i, t^{-}}^{2}\right)^{2} .
\end{aligned}
$$

Since $\int_{0}^{2 \pi}\left(1-\cos ^{4} \theta\right) \frac{d \theta}{2 \pi}=\frac{5}{8}$, from (13) we obtain $\partial_{t} h_{t} \leq-\frac{5}{8} h_{t}+C \frac{n}{N}$ (we have simply discarded the negative term with the indicator $\mathbf{1}_{[n, N)}(\xi)$ in $(\underline{13})$ ), and since $h_{0}=0$, the 
estimate for $\mathcal{W}_{2}^{2}$ follows from Gronwall's lemma:

$$
h_{t} \leq C\left(1-e^{-5 t / 8}\right) \frac{n}{N} \leq C \frac{n}{N}
$$

The estimate for $\mathcal{W}_{4}^{4}$ can be reduced to the previous one using an argument similar to the proof of [8, Corollary 3]: for $i \in\{1, \ldots, n\}$, call $S_{i, t}$ the event in which $U_{i, t}$ and $\tilde{U}_{i, t}$ have the same sign. On $S_{i, t}$ we have

$$
\left(U_{i, t}-\tilde{U}_{i, t}\right)^{4} \leq\left(U_{i, t}-\tilde{U}_{i, t}\right)^{2}\left(U_{i, t}+\tilde{U}_{i, t}\right)^{2}=\left(U_{i, t}^{2}-\tilde{U}_{i, t}^{2}\right)^{2}
$$

and then, using Hölder's inequality with $a=\frac{p}{p-4}$ and $b=p / 4$, we obtain

$$
\begin{aligned}
\mathbb{E}\left(U_{i, t}-\tilde{U}_{i, t}\right)^{4} & \leq \mathbb{E} \mathbf{1}_{S_{i, t}}\left(U_{i, t}^{2}-\tilde{U}_{i, t}^{2}\right)^{2}+\mathbb{E} \mathbf{1}_{S_{i, t}^{c}}\left(U_{i, t}-\tilde{U}_{i, t}\right)^{4} \\
& \leq \mathbb{E}\left(U_{i, t}^{2}-\tilde{U}_{i, t}^{2}\right)^{2}+\mathbb{P}\left(S_{i, t}^{c}\right)^{1 / a}\left[\mathbb{E}\left(U_{i, t}-\tilde{U}_{i, t}\right)^{4 b}\right]^{1 / b}
\end{aligned}
$$

The first term in the r.h.s. of this inequality is bounded by $C n / N$ thanks to (14), while the expectation in the second term is bounded uniformly on $t$ thanks to Lemma 4 . Also, we have $\mathbb{P}\left(S_{i, t}^{c}\right) \leq n /(2 N)$ : from (6) and (12) we see that when the processes $U_{i, t}$ and $\tilde{U}_{i, t}$ have a joint jump, they acquire the same sign (the one of $\cos \theta$ ), and form (5) and (11), it is easy to see that this occurs a proportion $1-n /(2 N)$ of the jumps on average. With all these, we get

$$
\mathcal{W}_{4}^{4}\left(\mathcal{L}^{n}\left(\mathbf{U}_{t}\right), f_{t}^{\otimes n}\right) \leq \mathbb{E} \frac{1}{n} \sum_{i=1}^{n}\left(U_{i, t}-\tilde{U}_{i, t}\right)^{4} \leq C\left(\frac{n}{N}\right)^{1 / a}
$$

which proves the estimate for $\mathcal{W}_{4}^{4}$.

To prove the following lemma, we will need some preliminaries. For a probability measure $\mu$ on $\mathbb{R}$, for any $q \geq 1$ and any $n \in \mathbb{N}$, define $\varepsilon_{q, n}(\mu):=\mathbb{E} \mathcal{W}_{q}^{q}(\overline{\mathbf{Z}}, \mu)$, where $\mathbf{Z}=$ $\left(Z_{1}, \ldots, Z_{n}\right)$ is an i.i.d. and $\mu$-distributed tuple. The best avaliable estimates for $\varepsilon_{q, n}(\mu)$ can be found in [7, Theorem 1]: if $\mu$ has finite $r$-moment for some $r>q, r \neq 2 q$, then there exists a constant $C$ depending only on $q$ and $r$ such that for $\eta=\min (1 / 2,1-q / r)$, it holds

$$
\varepsilon_{q, n}(\mu) \leq C \frac{\left(\int|x|^{r} \mu(d x)\right)^{q / r}}{n^{\eta}} .
$$

We will also need the following bound, which is a consequence of [6, Lemma 7]: given an exchangeable random vector $\mathbf{X} \in \mathbb{R}^{N}$ and a probability measure $\mu$ on $\mathbb{R}$, there exists a constant $C$, depending only on the $q$-moments of $\mu$ and $X_{1}$, such that for all $n \leq N$,

$$
\frac{1}{2^{q-1}} \mathbb{E} \mathcal{W}_{q}^{q}(\overline{\mathbf{X}}, \mu) \leq \mathcal{W}_{q}^{q}\left(\mathcal{L}^{n}(\mathbf{X}), \mu^{\otimes n}\right)+\varepsilon_{q, n}(\mu)+C \frac{n}{N}
$$

As a consequence of these estimates and Lemma 6, we have:

Lemma 7. Assume that $\int_{\mathbb{R}}|v|^{p} f_{0}(d v)<\infty$ for some $p>4, p \neq 8$. Then there exists a constant $C$ depending only on $p$ and $\int_{\mathbb{R}}|v|^{p} f_{0}(d v)$ such that for $\gamma=\min \left(\frac{1}{3}, \frac{p-4}{2 p-4}\right)$ and for all $t \geq 0$,

$$
\mathbb{E} \mathcal{W}_{2}^{2}\left(\overline{\mathbf{U}}_{t}^{(2)}, f_{t}^{(2)}\right) \leq \frac{C}{N^{\gamma}}
$$


and for $\tilde{\gamma}=\frac{p-4}{2 p} \mathbf{1}_{p<8}+\frac{p-4}{3 p-8} \mathbf{1}_{p>8}$,

$$
\mathbb{E} \mathcal{W}_{4}^{4}\left(\overline{\mathbf{U}}_{t}, f_{t}\right) \leq \frac{C}{N \tilde{\gamma}}
$$

Moreover, the same bounds hold with $\overline{\mathbf{U}}_{i, t}^{(2)}$ in place of $\overline{\mathbf{U}}_{t}^{(2)}$ and with $\overline{\mathbf{U}}_{i, t}$ in place of $\overline{\mathbf{U}}_{t}$, respectively.

Proof. Using the first part of Lemma 6 and (15)-(16) with $\mu=f_{t}^{(2)}, q=2$ and $r=p / 2$, we obtain $\mathbb{E} \mathcal{W}_{2}^{2}\left(\overline{\mathbf{U}}_{t}^{(2)}, f_{t}^{(2)}\right) \leq C\left[n^{-\eta}+n / N\right]$ for $\eta=\min (1 / 2,1-4 / p)(C$ depends on the $p / 2$ moments of $f_{t}^{(2)}$, which are controlled uniformly on $t$ thanks to Lemma 4). Taking $n=\left\lfloor N^{1 /(1+\eta)}\right\rfloor$ gives the estimate for $\mathcal{W}_{2}^{2}$. The estimate for $\mathcal{W}_{4}^{4}$ follows similarly: using the second part of Lemma 6 and (15)-(16) with $\mu=f_{t}, q=4$ and $r=p$, we obtain $\mathbb{E} \mathcal{W}_{4}^{4}\left(\overline{\mathbf{U}}_{t}, f_{t}\right) \leq C\left[n^{-\eta}+(n / N)^{1 / a}\right]$, for $a=\frac{p}{p-4}$ and the same $\eta=\min (1 / 2,1-4 / p)$. Taking $n=\left\lfloor N^{1 /(1+a \eta)}\right\rfloor$ gives the desired bound.

The estimates for $\overline{\mathbf{U}}_{i, t}^{(2)}$ and $\overline{\mathbf{U}}_{i, t}$ are obtained similarly.

We can now prove Theorem 1

Proof of Theorem 1. For some $i \in\{1, \ldots, N\}$ fixed, we will estimate the quantity $h_{t}:=$ $\mathbb{E}\left(V_{i, t}^{2}-U_{i, t}^{2}\right)^{2}$. Let us first shorten notation: call $V=V_{i, t^{-}}, V_{*}=V_{\mathbf{i}(\xi), t^{-}}, U=U_{i, t^{-}}$, $F=F_{i, t}\left(\mathbf{U}_{t^{-}}, \xi\right)$, and $U_{*}=U_{\mathbf{i}(\xi), t^{-}}$. From (4) and (6) , we have

$$
\begin{aligned}
d h_{t}=\mathbb{E} \int_{0}^{2 \pi} \int_{A_{i}}[ & \left.\left(V^{2}+V_{*}^{2}-U^{2}-F^{2}\right)^{2} \cos ^{4} \theta-\left(V^{2}-U^{2}\right)^{2}\right] \mathcal{N}_{i}(d t, d \theta, d \xi) \\
=\mathbb{E} \int_{0}^{2 \pi} \int_{A_{i}}[ & \left(\cos ^{4} \theta-1\right)\left(V^{2}-U^{2}\right)^{2} \\
& +\cos ^{4} \theta\left(V_{*}^{2}-U_{*}^{2}\right)^{2}+\cos ^{4} \theta\left(U_{*}^{2}-F^{2}\right)^{2} \\
& +2 \cos ^{4} \theta\left(V^{2}-U^{2}+V_{*}^{2}-U_{*}^{2}\right)\left(U_{*}^{2}-F^{2}\right) \\
& \left.+2 \cos ^{4} \theta\left(V^{2}-U^{2}\right)\left(V_{*}^{2}-U_{*}^{2}\right)\right] \frac{d t d \theta d \xi}{2 \pi(N-1)}
\end{aligned}
$$

Clearly $\mathbb{E} \int_{A_{i}}\left(V_{*}^{2}-U_{*}^{2}\right)^{2} \frac{d \xi}{N-1}=h_{t}$, by exchangeability. Thus, the first and second terms in the integral of (17) yield $-h_{t} d t \int_{0}^{2 \pi}\left(1-2 \cos ^{4} \theta\right) \frac{d \theta}{2 \pi}=-\frac{1}{4} h_{t} d t$. From (77), we have $\mathbb{E} \int_{A_{i}}\left(U_{*}^{2}-F^{2}\right)^{2} \frac{d \xi}{N-1}=\mathbb{E} \mathcal{W}_{2}^{2}\left(\overline{\mathbf{U}}_{i, t}^{(2)}, f_{t}^{(2)}\right) \leq C N^{-\gamma}$, thanks to Lemma 7. Using the CauchySchwarz inequality, the third and fourth terms in the integral of (17) are thus bounded above by $\left[C N^{-\gamma}+C h_{t}^{1 / 2} N^{-\gamma / 2}\right] d t$. For the remaining term, since $\frac{1}{N} \sum_{j} V_{j, t}^{2}=E_{N}$ for all $t \geq 0$ a.s., we have

$$
\begin{aligned}
& \mathbb{E}\left(V_{i, t}^{2}-U_{i, t}^{2}\right) \int_{A_{i}}\left(V_{\mathbf{i}(\xi), t}^{2}-U_{\mathbf{i}(\xi), t}^{2}\right) d \xi \\
& =\mathbb{E}\left(V_{i, t}^{2}-U_{i, t}^{2}\right)\left(-V_{i, t}^{2}+U_{i, t}^{2}+N E_{N}-\sum_{j=1}^{N} U_{j, t}^{2}\right) \\
& \leq-h_{t}+h_{t}^{1 / 2}\left[\mathbb{E}\left(\sum_{j=1}^{N}\left(U_{j, t}^{2}-\mathcal{E}\right)\right)^{2}\right]^{1 / 2}+N h_{t}^{1 / 2}\left[\mathbb{E}\left(E_{N}-\mathcal{E}\right)^{2}\right]^{1 / 2} \\
& =-h_{t}+h_{t}^{1 / 2}\left[N \operatorname{var}\left(U_{i, t}^{2}\right)+N(N-1) \operatorname{cov}\left(U_{i, t}^{2}, U_{j, t}^{2}\right)\right]^{1 / 2}+N h_{t}^{1 / 2} B_{N}^{1 / 2},
\end{aligned}
$$


where in the last line $j \neq i$ is any fixed index, and $B_{N}:=\mathbb{E}\left(E_{N}-\mathcal{E}\right)^{2}$. Thanks to lemmas 4 and 5, the latter is bounded by $-h_{t}+C h_{t}^{1 / 2} N^{1 / 2}+N h_{t}^{1 / 2} B_{N}^{1 / 2}$; thus, the fifth term of (17) is controlled by $-\frac{3}{4(N-1)} h_{t} d t+C h_{t}^{1 / 2}\left[N^{-1 / 2}+B_{N}^{1 / 2}\right] d t$. Gathering all these estimates, we get from (17)

$$
\begin{aligned}
\partial_{t} h_{t} & \leq-\left(\frac{1}{4}+\frac{3}{4(N-1)}\right) h_{t}+C h_{t}^{1 / 2}\left[N^{-\gamma / 2}+N^{-1 / 2}+B_{N}^{1 / 2}\right]+C N^{-\gamma} \\
& \leq-\lambda_{N} h_{t}+C h_{t}^{1 / 2}\left[N^{-\gamma / 2}+B_{N}^{1 / 2}\right]+C N^{-\gamma} .
\end{aligned}
$$

Using a version of Gronwall's lemma (see for instance [1, Lemma 4.1.8]), we obtain

$$
h_{t} \leq C e^{-\lambda_{N} t} h_{0}+C N^{-\gamma}+C B_{N} .
$$

Finally, note that $\mathbb{E} \mathcal{W}_{2}^{2}\left(\overline{\mathbf{V}}_{t}^{(2)}, f_{t}^{(2)}\right) \leq 2 \mathbb{E} \mathcal{W}_{2}^{2}\left(\overline{\mathbf{V}}_{t}^{(2)}, \overline{\mathbf{U}}_{t}^{(2)}\right)+2 \mathbb{E} \mathcal{W}_{2}^{2}\left(\overline{\mathbf{U}}_{t}^{(2)}, f_{t}^{(2)}\right)$, and, since $\mathbb{E} \mathcal{W}_{2}^{2}\left(\overline{\mathbf{V}}_{t}^{(2)}, \overline{\mathbf{U}}_{t}^{(2)}\right) \leq \mathbb{E} \frac{1}{N} \sum_{j}\left(V_{j, t}^{2}-U_{j, t}^{2}\right)^{2}=h_{t}$ by exchangeability, the conclusion follows from (18), the first part of Lemma 7 , and choosing $\left(\mathbf{V}_{0}, \mathbf{U}_{0}\right)$ as an optimal coupling with respect to the cost $\left(x^{2}-y^{2}\right)^{2}$, so $h_{0}=\mathcal{W}_{2}^{2}\left(\mathcal{L}\left(\mathbf{V}_{0}^{(2)}\right),\left(f_{0}^{(2)}\right)^{\otimes N}\right)$.

Proof of Corollary 2. The argument is the same as in the proof of [8, Corollary 3], and we repeat it here for convenience of the reader. From (44) and (6), it is clear that $V_{i, t}$ and $U_{i, t}$ have the same $\operatorname{sign}($ the one of $\cos \theta$ ) after the first jump. And if they have the same sign, then

$$
\left(V_{i, t}-U_{i, t}\right)^{4} \leq\left(V_{i, t}-U_{i, t}\right)^{2}\left(V_{i, t}+U_{i, t}\right)^{2}=\left(V_{i, t}^{2}-U_{i, t}^{2}\right)^{2} .
$$

Call $\tau_{i}$ the time of the first jump of $V_{i, t}$. Then

$$
\begin{aligned}
\mathbb{E}\left(V_{i, t}-U_{i, t}\right)^{4} & \leq \mathbb{E} \mathbf{1}_{\left\{\tau_{i} \leq t\right\}}\left(V_{i, t}^{2}-U_{i, t}^{2}\right)^{2}+\mathbb{E} \mathbf{1}_{\left\{\tau_{i}>t\right\}}\left(V_{i, t}-U_{i, t}\right)^{4} \\
& \leq \mathbb{E}\left(V_{i, t}^{2}-U_{i, t}^{2}\right)^{2}+\mathbb{E} \mathbf{1}_{\left\{\tau_{i}>t\right\}}\left(V_{i, 0}-U_{i, 0}\right)^{4} .
\end{aligned}
$$

For the second term we use the fact that $\tau_{i}$ is independent of $\left(V_{i, 0}, U_{i, 0}\right)$ and has exponential distribution with parameter 1 , which gives $e^{-t} \mathbb{E}\left(V_{i, 0}-U_{i, 0}\right)^{4}$. For the first term we simply use (18). This yields

$$
\begin{aligned}
\mathbb{E} \frac{1}{N} \sum_{i}\left(V_{i, t}-U_{i, t}\right)^{4} \leq & C N^{-\gamma}+C e^{-\lambda_{N} t} \mathbb{E} \frac{1}{N} \sum_{i}\left(V_{i, 0}^{2}-U_{i, 0}^{2}\right)^{2} \\
& +C \mathbb{E}\left(E_{N}-\mathcal{E}\right)^{2}+C e^{-t} \mathbb{E} \frac{1}{N} \sum_{i}\left(V_{i, 0}-U_{i, 0}\right)^{4}
\end{aligned}
$$

Finally, we have $\mathbb{E} \mathcal{W}_{4}^{4}\left(\overline{\mathbf{V}}_{t}, f_{t}\right) \leq C \mathbb{E} \mathcal{W}_{4}^{4}\left(\overline{\mathbf{V}}_{t}, \overline{\mathbf{U}}_{t}\right)+C \mathbb{E} \mathcal{W}_{4}^{4}\left(\overline{\mathbf{U}}_{t}, f_{t}\right)$, and the result follows since the first term is bounded above by $C \mathbb{E} \frac{1}{N} \sum_{i}\left(V_{i, t}-U_{i, t}\right)^{4}$ and using the second part of Lemma 7 on the second term (recall that $\tilde{\gamma}<\gamma$ ).

To prove Theorem 3, we will need the results of [8]. They provide exponential contraction rates in $\mathcal{W}_{4}^{4}$ for both the particle system and the nonlinear process, which in turn imply contraction in $\mathcal{W}_{2}^{2}$. More specifically: assuming $\sup _{N} \mathbb{E} V_{1,0}^{4}<\infty$ and $\int_{\mathbb{R}} v^{4} f_{0}(d v)<\infty$, one has for some $\alpha>0$

$$
\mathcal{W}_{2}^{2}\left(\mathcal{L}\left(\mathbf{V}_{t}\right), \mathcal{U}_{N}\right) \leq C e^{-\alpha t} \quad \text { and } \quad \mathcal{W}_{2}^{2}\left(f_{t}, f_{\infty}\right) \leq C e^{-\alpha t}
$$

where $\mathcal{U}_{N}$ and $f_{\infty}$ are the stationary distributions for the particle system and nonlinear process, respectively. Namely, $\mathcal{U}_{N}$ is the uniform distribution on the sphere $\left\{\mathbf{x} \in \mathbb{R}^{N}\right.$ : 
$\left.\frac{1}{N} \sum_{i} x_{i}^{2}=r^{2}\right\}$ with $r^{2}$ chosen randomly with the same law as $E_{N}=\frac{1}{N} \sum_{i} V_{i, 0}^{2}$, and $f_{\infty}$ is the Gaussian distribution with mean 0 and variance $\mathcal{E}=\int v^{2} f_{0}(d v)$ (note that, although the results of [8] are stated in the case $E_{N}=1$ a.s., it is easy to generalize them to the case of particle systems starting a.s. with the same random energy).

Also, it is easy to verify that

$$
\mathcal{W}_{2}^{2}\left(\mathcal{U}_{N}, f_{\infty}^{\otimes N}\right) \leq C N^{-1 / 2}+C \mathcal{W}_{2}^{2}\left(\mathcal{L}\left(\mathbf{V}_{0}\right), f_{0}^{\otimes N}\right) .
$$

Indeed, given a random vector $\mathbf{Z}=\left(Z_{1}, \ldots, Z_{N}\right)$ with law $f_{\infty}^{\otimes N}$ independent of $\mathbf{V}_{0}$, call $Q^{2}=\frac{1}{N} \sum_{i=1}^{N} Z_{i}^{2}$ and define $Y_{i}=E_{N}^{1 / 2} Z_{i} / Q$, so that $\mathbf{Y}=\left(Y_{1}, \ldots, Y_{N}\right)$ has distribution $\mathcal{U}_{N}$ thanks to the fact that $f_{\infty}^{\otimes N}$ is rotation invariant. A straightforward computation shows that $\frac{1}{N} \sum_{i}\left(Z_{i}-Y_{i}\right)^{2}=\left(Q-E_{N}^{1 / 2}\right)^{2} \leq 2\left(Q-\mathcal{E}^{1 / 2}\right)^{2}+2\left(E_{N}^{1 / 2}-\mathcal{E}^{1 / 2}\right)^{2}$, which is bounded above by $2 \mathcal{W}_{2}^{2}\left(\overline{\mathbf{Z}}, f_{\infty}\right)+2 \mathcal{W}_{2}^{2}\left(\overline{\mathbf{V}}_{0}, f_{0}\right)$, since $\int v^{2} f_{\infty}(d v)=\int v^{2} f_{0}(d v)=\mathcal{E}$ (in general, for measures $\mu$ and $\nu$ on $\mathbb{R}$ with $Q_{\mu}^{2}=\int x^{2} \mu(d x)$, one has for any $X \sim \mu$ and $\left.\tilde{X} \sim \nu: \mathbb{E}(X-\tilde{X})^{2} \geq Q_{\mu}^{2}+Q_{\nu}^{2}-2 Q_{\mu} Q_{\nu}=\left(Q_{\mu}-Q_{\nu}\right)^{2}\right)$. This coupling gives $\mathcal{W}_{2}^{2}\left(\mathcal{U}_{N}, f_{\infty}^{\otimes N}\right) \leq \mathbb{E} \frac{1}{N} \sum_{i}\left(Z_{i}-Y_{i}\right)^{2} \leq 2 \mathbb{E} \mathcal{W}_{2}^{2}\left(\overline{\mathbf{Z}}, f_{\infty}\right)+4 \mathbb{E} \mathcal{W}_{2}^{2}\left(\overline{\mathbf{V}}_{0}, \overline{\mathbf{U}}_{0}\right)+4 \mathbb{E} \mathcal{W}_{2}^{2}\left(\overline{\mathbf{U}}_{0}, f_{0}\right)$, where the first and third terms are controlled by $C N^{-1 / 2}$ thanks to (15), and the second term is controlled by $4 \mathbb{E} \frac{1}{N} \sum_{i}\left(V_{i, 0}-U_{i, 0}\right)^{2}=4 \mathcal{W}_{2}^{2}\left(\mathcal{L}\left(\mathbf{V}_{0}\right), f_{0}^{\otimes N}\right)$, this time choosing the initial conditions $\left(\mathbf{V}_{0}, \mathbf{U}_{0}\right)$ as an optimal coupling with respect to the usual quadratic cost $(x-y)^{2}$.

We are now ready to prove Theorem 3 :

Proof of Theorem [3. The argument combines the contraction results of [8] and the propagation of chaos results of [6]. Clearly,

$$
\begin{aligned}
& \mathbb{E} \mathcal{W}_{2}^{2}\left(\overline{\mathbf{V}}_{t}, f_{t}\right) \\
& \leq C \mathbb{E}\left[\mathcal{W}_{2}^{2}\left(\overline{\mathbf{V}}_{t}, \overline{\mathbf{V}}_{\infty}\right)+\mathcal{W}_{2}^{2}\left(\overline{\mathbf{V}}_{\infty}, \overline{\mathbf{Z}}_{\infty}\right)+\mathcal{W}_{2}^{2}\left(\overline{\mathbf{Z}}_{\infty}, f_{\infty}\right)+\mathcal{W}_{2}^{2}\left(f_{\infty}, f_{t}\right)\right]
\end{aligned}
$$

Here $\mathbf{V}_{\infty}$ is a random vector on $\mathbb{R}^{N}$ with law $\mathcal{U}_{N}$, which is also optimally coupled to $\mathbf{V}_{t}$ with respect to the quadratic cost, so $\mathbb{E} \mathcal{W}_{2}^{2}\left(\overline{\mathbf{V}}_{t}, \overline{\mathbf{V}}_{\infty}\right) \leq \mathbb{E} \frac{1}{N} \sum_{i}\left(V_{i, t}-V_{i, \infty}\right)^{2}=$ $\mathcal{W}_{2}^{2}\left(\mathcal{L}\left(\mathbf{V}_{t}\right), \mathcal{L}\left(\mathbf{V}_{\infty}\right)\right)$. Thus, the first and fourth term are bounded by $C e^{-\alpha t}$, thanks to (19). Also, we have chosen $\mathbf{Z}_{\infty}$ with law $f_{\infty}^{\otimes N}$ and being optimally coupled to $\mathbf{V}_{\infty}$, so for the second term of (21) we have $\mathbb{E} \mathcal{W}_{2}^{2}\left(\overline{\mathbf{V}}_{\infty}, \overline{\mathbf{Z}}_{\infty}\right) \leq \mathcal{W}_{2}^{2}\left(\mathcal{U}_{N}, f_{\infty}^{\otimes N}\right)$, which is controlled using (20). The third term is controlled by $C N^{-1 / 2}$, thanks to (15). With all these estimates, we obtain from (21):

$$
\mathbb{E} \mathcal{W}_{2}^{2}\left(\overline{\mathbf{V}}_{t}, f_{t}\right) \leq C e^{-\alpha t}+C \mathcal{W}_{2}^{2}\left(\mathcal{L}\left(\mathbf{V}_{0}\right), f_{0}^{\otimes N}\right)+C N^{-1 / 3}
$$

for some $\alpha>0$. On the other hand, from [6, Theorem 1] we have

$$
\mathbb{E} \mathcal{W}_{2}^{2}\left(\overline{\mathbf{V}}_{t}, f_{t}\right) \leq C \mathcal{W}_{2}^{2}\left(\mathcal{L}\left(\mathbf{V}_{0}\right), f_{0}^{\otimes N}\right)+C(1+t)^{2} N^{-1 / 3}
$$

(In [6] the initial distribution of the particle system was chosen as $f_{0}^{\otimes N}$, but the extension to any exchangeable initial condition is straightforward). Finally, the result is obtained from (22) and (23) adjusting $t$ and $N$ conveniently: take $t_{*}=\frac{\log N}{3 \alpha}$, so (22) yields $\mathbb{E} \mathcal{W}_{2}^{2}\left(\overline{\mathbf{V}}_{t}, f_{t}\right) \leq C \mathcal{W}_{2}^{2}\left(\mathcal{L}\left(\mathbf{V}_{0}\right), f_{0}^{\otimes N}\right)+C N^{-1 / 3}$ for $t \geq t_{*}$, whereas (23) gives $\mathbb{E} \mathcal{W}_{2}^{2}\left(\overline{\mathbf{V}}_{t}, f_{t}\right) \leq C \mathcal{W}_{2}^{2}\left(\mathcal{L}\left(\mathbf{V}_{0}\right), f_{0}^{\otimes N}\right)+C N^{-1 / 3} \log ^{2} N$ for $t \leq t_{*}$. The result follows.

Acknowledgements. The author thanks Joaquin Fontbona and Jean-François Jabir for very useful suggestions and corrections of earlier versions of this manuscript. 


\section{References}

[1] Luigi Ambrosio, Nicola Gigli, and Giuseppe Savaré. Gradient flows in metric spaces and in the space of probability measures. Lectures in Mathematics ETH Zürich. Birkhäuser Verlag, Basel, second edition, 2008.

[2] E.A. Carlen, M.C. Carvalho, and M. Loss. Determination of the spectral gap for Kac's master equation and related stochastic evolution. Acta Mathematica, 191(1):154, 2003.

[3] Eric Carlen, M. C. Carvalho, and Michael Loss. Many-body aspects of approach to equilibrium. In Journées "Équations aux Dérivées Partielles" (La Chapelle sur Erdre, 2000), pages Exp. No. XI, 12. Univ. Nantes, Nantes, 2000.

[4] Eric A. Carlen, Maria C. Carvalho, Jonathan Le Roux, Michael Loss, and Cédric Villani. Entropy and chaos in the Kac model. Kinet. Relat. Models, 3(1):85-122, 2010.

[5] Kleber Carrapatoso and Amit Einav. Chaos and entropic chaos in Kac's model without high moments. Electron. J. Probab., 18:no. 78, 38, 2013.

[6] Roberto Cortez and Joaquin Fontbona. Quantitative propagation of chaos for generalized Kac particle systems. Ann. Appl. Probab., 26(2):892-916, 2016.

[7] Nicolas Fournier and Arnaud Guillin. On the rate of convergence in Wasserstein distance of the empirical measure. Probability Theory and Related Fields, 162(34):707-738, 2015.

[8] Maxime Hauray. Uniform Contractivity in Wasserstein Metric for the Original 1D Kac's Model. J. Stat. Phys., 162(6):1566-1570, 2016.

[9] M. Kac. Foundations of kinetic theory. In Proceedings of the Third Berkeley Symposium on Mathematical Statistics and Probability, 1954-1955, vol. III, pages 171-197, Berkeley and Los Angeles, 1956. University of California Press.

[10] Stéphane Mischler and Clément Mouhot. Kac's program in kinetic theory. Invent. Math., 193(1):1-147, 2013.

[11] Alain-Sol Sznitman. Topics in propagation of chaos. In École d'Été de Probabilités de Saint-Flour XIX-1989, volume 1464 of Lecture Notes in Math., pages 165-251. Springer, Berlin, 1991.

[12] Hiroshi Tanaka. Probabilistic treatment of the Boltzmann equation of Maxwellian molecules. Z. Wahrsch. Verw. Gebiete, 46(1):67-105, 1978/79.

[13] Cédric Villani. Optimal transport, old and new, volume 338 of Grundlehren der Mathematischen Wissenschaften [Fundamental Principles of Mathematical Sciences]. Springer-Verlag, Berlin, 2009. 